\title{
Hybrid Concatenated LDPC Codes with LTE Modulation Schemes
}

\author{
Mohanad Alfiras ${ }^{1}$ \\ Communication and networks \\ Engineering, Gulf University, \\ Manama, Kingdom of Bahrain
}

\author{
Wael A. H. Hadi ${ }^{2}$, Amjad Ali Jassim \\ Communication Engineering Department \\ Engineering college, Al-Technology University \\ Baghdad, Iraq
}

\begin{abstract}
In a communication system, the LDPC code is considered as a good performance error correcting code which reaches near Shannon limit. In this paper a hybrid LDPC code is proposed, the hybrid term here refers to the serial concatenation of parallel LDPC codes group and a single serial LDPC code. The outer two parallel LDPC codes encoder represents outer encoder where the single LDPC encoder represents the inner encoder. This study also emphases on the performance of a hybrid coding system in consideration with three modulation schemes. The modulation schemes include quadrature phase shift keying (QPSK) and two types of quadrature amplitude modulation; 16QAM and 64-QAM. These modulation schemes are selected due to their importance in modern communication applications, such as long term evolution (LTE); such schemes are the standard modulation schemes used with LTE system. This study investigates different $L D P C$ code rates such as $1 / 2$ and $1 / 3$ and simulates the AWGN communication channel using MATLAB. The simulation results show improvement in bit error rate (BER) when using 1/3 LDPC code rate in the designed system rather than $1 / 2$, but it also increases the system complexity. In the end, all simulation results, the comparison between different cases of LDPC code rates and system performance are summarized.
\end{abstract}

Keywords-Coding; modulation; Hybrid; concatenation; low density parity check

\section{INTRODUCTION}

G. David Forny introduced the concept of concatenated codes in 1965 as a method to improve system code performance [1]. Earlier to this, Gallager in his Ph.D. dissertation at M.I.T invented LDPC codes in 1960 [2]. This paper focuses on the concept that uses the LDPC codes concatenation as an error correction code to improve communication system performance against errors raised during signal transmission through the noisy channel. The code concatenation improves the performance of the error correction code as a group against transmission errors. The simplest form of code concatenation is observed in serial concatenation between two error correction codes. The codes that are involved in serial concatenation can be of different or same type. For example, serial concatenation between two convolutional encoders [3] or between two different error correction codes, such as, read Solomon code and convolutional encoder [4] and between read Solomon code and LDPC code [5]. The error correction code concatenation also has another form which consists of parallel concatenation between two identical error correction codes [6]. For example, the parallel concatenation between two convolutional encoders forms a well-known Turbo code. The concatenation between two parallel convolutional encoders offers the significant BER Turbo code performance, but with increased decoder complexity. The decoder uses complicated algorithms to calculate decoding frame estimation such as SOVA and BCJR algorithms [7]. LDPC codes can be used with the same concept of code concatenation. Therefore, researchers focus on using serial concatenation of LDPC codes of the same code type and rate [8]. In order to form a serially concatenated codes, consider concatenation between LDPC codes of different types as compatible pairs [9]. In order to achieve that, the inner LDPC encoder takes code rates which are fitted in data rate with outer LDPC encoder. This strategy takes benefit of increasing only the inner LDPC encoder size and reduce the system complexity as compared when using two large size LDPC codes. Also, parallel concatenation is applicable for LDPC codes using two identical LDPC codes with a simple modification in the receiver to avoid increasing system decoder complexity [10]. This modification includes taking the sum of the two LDPC decoders that are based on bit flipping algorithm [11]. In many communication applications such as deep space communication systems, there is a need for accurate BER performance against raised communication errors rather than the system complexity [12]. Therefore, the system complexity could be acceptable in some communication applications where the actual goal of the system is to achieve a good BER performance [13]. This work focus on using short length irregular LDPC codes connected in Hybrid form, the term Hybrid concatenation used here refers to two types of concatenated codes, parallel concatenation between two identical LDPC encoders and serially concatenated with inner LDPC encoder. The concatenation strategy should be carefully designed to get better system performance and low system complexity as much as possible. So, different code rates and different modulation schemes are investigated by comparing different system designs.

\section{PROPOSED System TRANSMitTeR}

The proposed hybrid system transmitter consists of three main stages. First, represented by identical irregular LDPC codes connected in parallel to construct outer encoder. Second, the produced code words from two LDPC encoder are multiplexed to prepare input for the next stage of inner encoder LDPC with length equal to twice individual outer LDPC code. The inner code input frame length will be two times the output of a single LDPC code used to construct the outer parallel 
group. Third, the modulation scheme such as QPSK, 16-QAM or 64-QAM considered in modulation stage $[14,15]$ as LTE standard modulation schemes. The system transmitter is shown in Fig. 1.

In order to process the input data, the Hybrid system transmitter starts by the LDPC1 and LDPC2 encoders which are designed to be identical. Each code of data rate equal to $1 / \mathrm{n}_{1}$. There is an interleaver between these parallel LDPC1 and LDPC2 encoders which is denoted by $\pi_{1}$ in Fig. 1. The interleaver rearrange the order of input data frame to construct a new code word which differs from the code word of LDPC1 encoder. The interleaver could be random or another type. Here, the main usage of interleaver is to overcome the effects of burst errors, if found in the received codeword and change it to suppurated errors that could be handled and corrected by LDPC decoder. Each LDPC1 and LDPC2 encoder of code rate

$\mathrm{k} / \mathrm{n}_{1}=\mathrm{k} / \mathrm{n}_{2}=\mathrm{k} / \mathrm{n}$

Where ' $k$ ' represents the length of the input data frame, and the ' $n$ ' represents the length of the produced code word. Therefore, after the parallel encoding process and multiplexer, it produces a codeword of length

$1 /\left(2 \times \mathrm{n}_{1}\right)=1 /\left(2 \times \mathrm{n}_{2}\right)=1 /(2 \times \mathrm{n})$

Inner LDPC encoder is designed to be of different length, not the same as LDPC codes which are used with an outer parallel group. The length of the input data which inner LDPC takes is the same as the length of the produced codeword by the outer parallel group. Then the inner LDPC code rate $1 / n_{3}$, in terms of input is

$\mathrm{R}=\left(2 \times \mathrm{n}_{1}\right) /\left(2 \times \mathrm{n}_{1} \times \mathrm{n}_{3}\right)$

The symbol $\pi_{2}$ in system stands for random interleaver used to enhance system performance against burst errors. In general, for LDPC codes, the larger length LDPC encoder gives the best performance as compared with shorter ones. However, larger LDPC code length means more complex LDPC decoder in system receiver. The inner LDPC3 will be an effective inner encoder, without using a larger encoder.

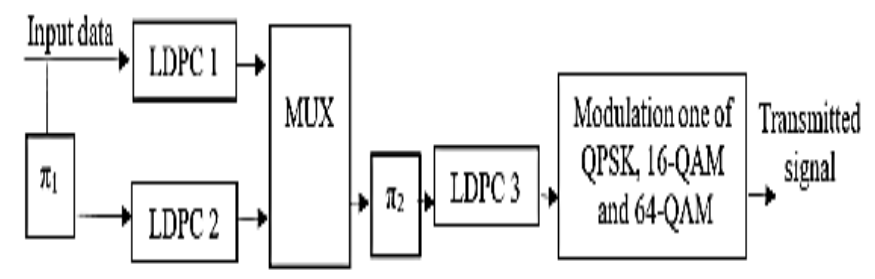

Fig. 1. Hybrid LDPC Code System Transmitter.

\section{PROPOSED SYSTEM RECEIVER}

The hybrid system receiver starts with the demodulation process which is the same as the modulation scheme used in the system transmitter. The decoding process starts in reciprocal order of the transmitter encoder process. It starts by decoding LDPC3 which represent inner encoder of the transmitter. This decoder uses the LLR (Log Likelihood Ratio algorithm) [16]. The LDPC decoder gives estimation output which is denoted by $\hat{E}_{3}$. The decoding algorithm LLR is described as follow [16]:
The input to the LDPC decoder is the log-likelihood ratio (LLR), $L\left(c_{i}\right)$, which is defined by

$L\left(c_{i}\right)=\log \left(\frac{\operatorname{Pr}\left(c_{i}=0 \mid \text { channel output for } c i\right)}{\operatorname{Pr}\left(c_{i}=1 \mid \text { channel output for ci }\right)}\right)$

Where $c_{i}$ is an ith bit of the transmitted codeword $c$. There are three key variables in the algorithm: $L\left(r_{j i}\right), L\left(q_{i j}\right)$, and $L\left(Q_{i}\right)$. $L\left(q_{i j}\right)$ is initialized as $L\left(q_{i j}\right)=L\left(c_{i}\right)$. For each iteration, update $L\left(r_{j i}\right), L\left(q_{i j}\right)$, and $L\left(Q_{i}\right)$ using the following set of equations [16]:

$L\left(r_{j i}\right)=2 \operatorname{atanh}\left(\prod_{i^{\prime} \in V_{j \backslash i}} \tanh \left(\frac{1}{2} L\left(q_{i^{\prime} j}\right)\right)\right)$

$L\left(q_{i j}\right)=L\left(c_{i}\right)+\sum_{j^{\prime} \in c_{i} \backslash j} L\left(r_{j^{\prime} i}\right)$

$L\left(Q_{i}\right)=L\left(c_{i}\right)+\sum_{j^{\prime} \in c_{i}} L\left(r_{j^{\prime} i}\right)$

$\hat{\mathrm{E}} 3$ represents the estimated output from $\mathrm{LDPC}_{3}$, which is passed to de-multiplexer to redirect $\hat{\mathrm{E}}_{3}$ into two groups which are inputs for LDPC decoder one and LDPC decoder two, respectively. The LDPC decoders of one and two use the same described decoding algorithm LLR. Such a process at the end produce another two estimations denoted by $\hat{E}_{1}$ and $\hat{E}_{2}$ refers to each decoder of the parallel group. The two estimations then summed before the decision. The decision represents the final receiver stage to produce received data. Fig. 2 shows the proposed system receiver.

Where the symbol $\pi^{-1}$ refers to random de-interleaver.

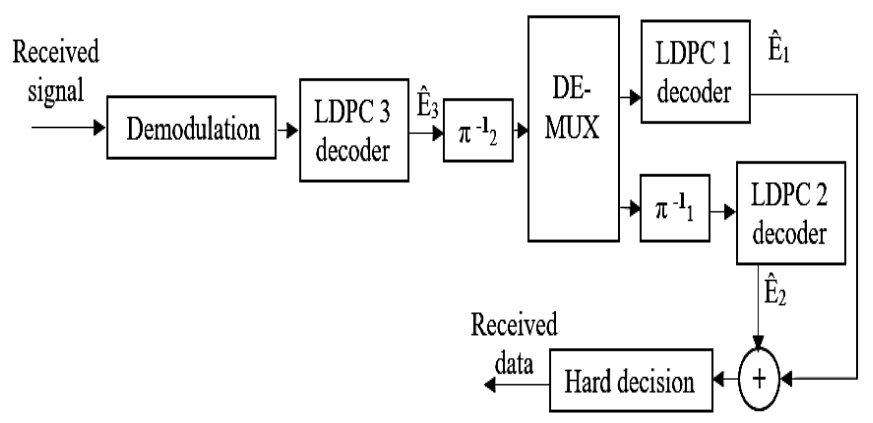

Fig. 2. Proposed Hybrid System Receiver.

\section{SIMULATION PARAMETERS}

The simulation includes the generation of two sets of irregular LDPC codes. First of rate $1 / 2$ and second of rate $1 / 3$. Table I and Table II summarize the description where the code is described by $\mathrm{C}_{\mathrm{b}}(\mathrm{N}, \mathrm{K}), \mathrm{N}$ codeword length, and $\mathrm{K}$ input data length.

The simulation takes in consideration of multiple hybrid system designs; it discusses the increase in the length of generated irregular LDPC codes and also designs the hybrid system with two LDPC code rates, first represented by rate $1 / 2$ and the second of rate $1 / 3$. It gives us more sense about increasing LDPC code rate and length and its effect in system BER performance. 
TABLE I. LDPC CODES RATE $1 / 2$

\begin{tabular}{|l|l|l|l|}
\hline \multirow{2}{*}{ Design } & \multicolumn{2}{|l|}{ Outer encoder parallel group } & Inner encoder \\
\cline { 2 - 4 } & $\mathrm{LDPC}$ & $\mathrm{LDPC} 2$ & LDPC3 \\
\hline Case 1 & $\mathrm{C}_{\mathrm{b}}(48,24)$ & $\mathrm{C}_{\mathrm{b}}(48,24)$ & $\mathrm{C}_{\mathrm{b}}(192,96)$ \\
\hline Case 2 & $\mathrm{C}_{\mathrm{b}}(96,48)$ & $\mathrm{C}_{\mathrm{b}}(96,48)$ & $\mathrm{C}_{\mathrm{b}}(384,192)$ \\
\hline Case 3 & $\mathrm{C}_{\mathrm{b}}(144,72)$ & $\mathrm{C}_{\mathrm{b}}(144,72)$ & $\mathrm{C}_{\mathrm{b}}(576,288)$ \\
\hline Case 4 & $\mathrm{C}_{\mathrm{b}}(192,96)$ & $\mathrm{C}_{\mathrm{b}}(192,96)$ & $\mathrm{C}_{\mathrm{b}}(768,384)$ \\
\hline Case 5 & $\mathrm{C}_{\mathrm{b}}(240,120)$ & $\mathrm{C}_{\mathrm{b}}(240,120)$ & $\mathrm{C}_{\mathrm{b}}(960,480)$ \\
\hline
\end{tabular}

TABLE II. LDPC CODES RATE $1 / 3$

\begin{tabular}{|l|l|l|l|}
\hline \multirow{2}{*}{ Design } & \multicolumn{2}{|l|}{ Outer encoder parallel group } & Inner encoder \\
\cline { 2 - 4 } & $\mathrm{LDPC} 1$ & $\mathrm{LDPC} 2$ & $\mathrm{LDPC} 3$ \\
\hline Case 1 & $\mathrm{C}_{\mathrm{b}}(72,24)$ & $\mathrm{C}_{\mathrm{b}}(72,24)$ & $\mathrm{C}_{\mathrm{b}}(432,144)$ \\
\hline Case 2 & $\mathrm{C}_{\mathrm{b}}(144,48)$ & $\mathrm{C}_{\mathrm{b}}(144,48)$ & $\mathrm{C}_{\mathrm{b}}(864,288)$ \\
\hline Case 3 & $\mathrm{C}_{\mathrm{b}}(216,72)$ & $\mathrm{C}_{\mathrm{b}}(216,72)$ & $\mathrm{C}_{\mathrm{b}}(1296,432)$ \\
\hline Case 4 & $\mathrm{C}_{\mathrm{b}}(288,96)$ & $\mathrm{C}_{\mathrm{b}}(288,96)$ & $\mathrm{C}_{\mathrm{b}}(1728,576)$ \\
\hline Case 5 & $\mathrm{C}_{\mathrm{b}}(360,120)$ & $\mathrm{C}_{\mathrm{b}}(360,120)$ & $\mathrm{C}_{\mathrm{b}}(2160,720)$ \\
\hline
\end{tabular}

\section{Simulation Results}

The proposed system simulation includes the cases and their corresponding generated irregular LDPC codes. It splits the system performance as BER vs. SNR into two groups depending on the LDPC code rate. In each case, a modulation scheme is selected from three types of QPSK which are used for low data rate while its symbol consists of two bits; 64QAM for high data rate with good quality SNR where each symbol consisted of 6 bits and 16-QAM with 4 bits per symbol. These modulation schemes are used as a standard with LTE application [4]. Fig. 3 to 8 shows simulation results, respectively.

The simulation result values (BER) compares different system parameters listed in Table III and Table IV, respectively.

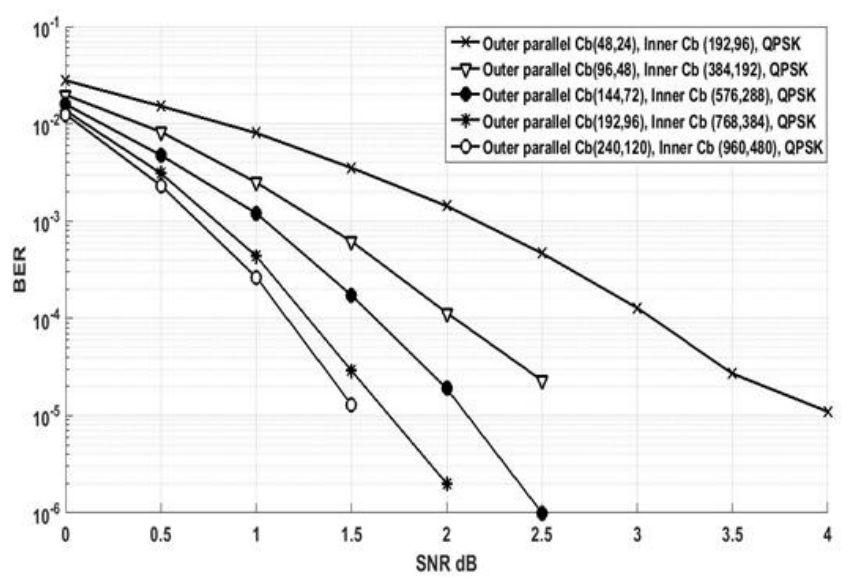

Fig. 3. LDPC Code Rate 1/2, QPSK.

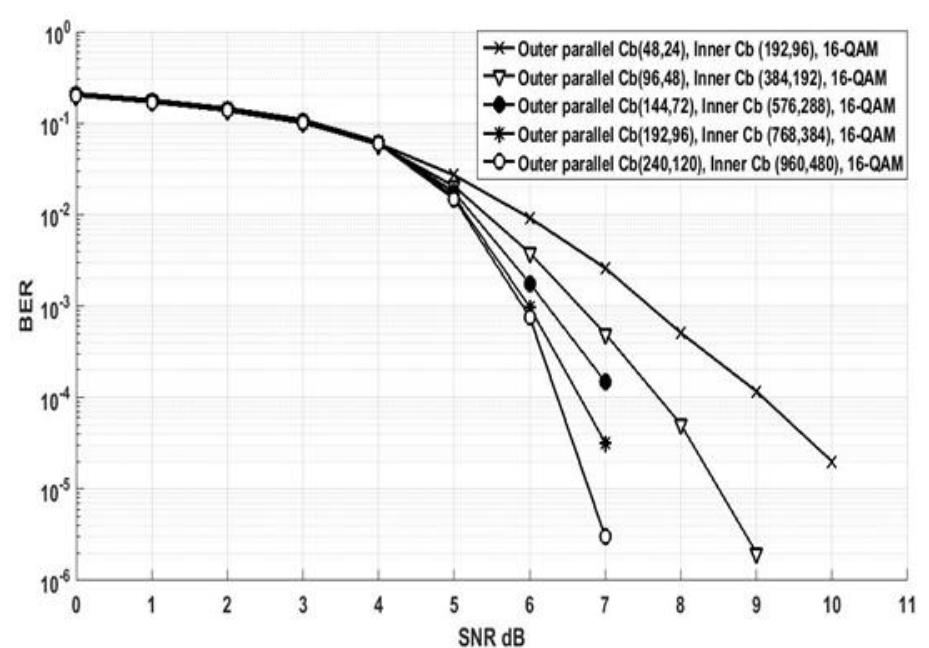

Fig. 4. LDPC Code Rate 1/2, 16-QAM.

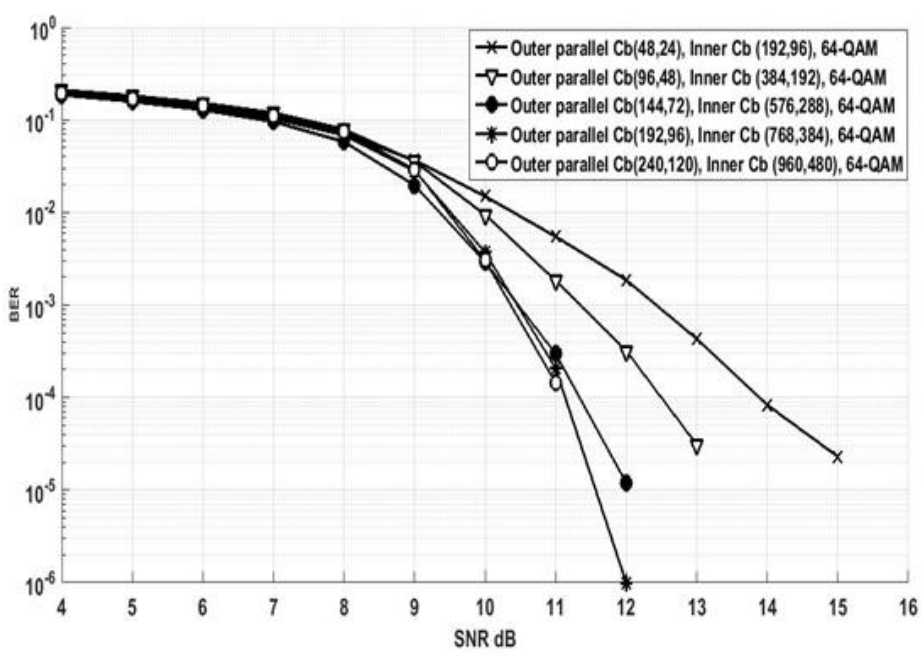

Fig. 5. LDPC Code Rate 1/2, 64-QAM.

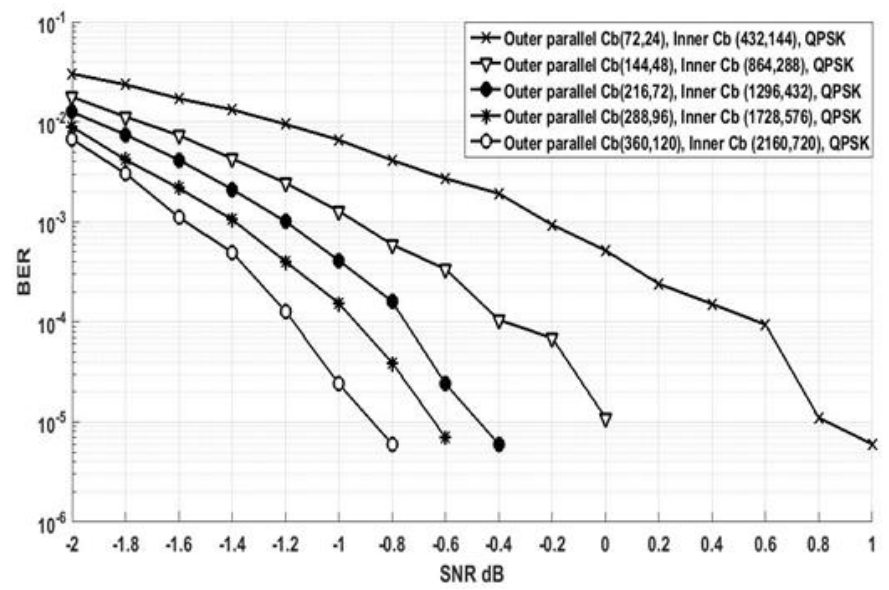

Fig. 6. LDPC Code Rate 1/3, QPSK. 


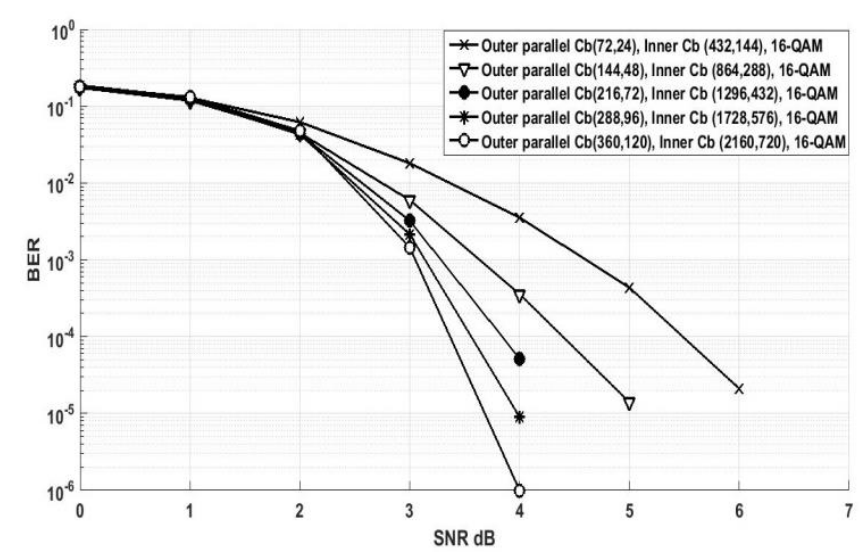

Fig. 7. LDPC Code Rate 1/3, 16-QAM.

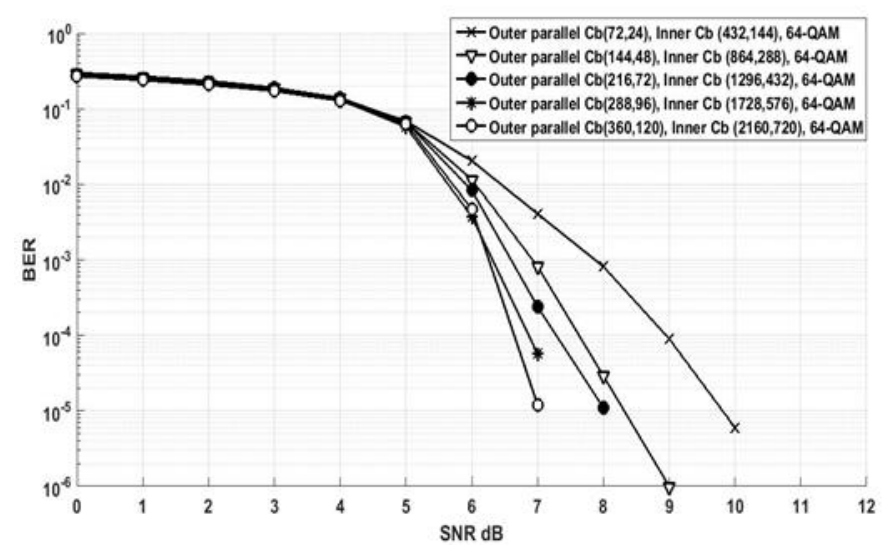

Fig. 8. LDPC Code Rate 1/3, 64-QAM.

TABLE III. ShOWS SimUlation RESUlTS IN COMPARISON TO HYBRID SYSTEM LDPC RATE 1/2

\begin{tabular}{|c|c|c|c|c|}
\hline $\begin{array}{c}\text { Modulation } \\
\text { type }\end{array}$ & $\begin{array}{c}\text { Outer Parallel } \\
\text { Two LDPC Codes }\end{array}$ & $\begin{array}{c}\text { Inner LDPC } \\
\text { code }\end{array}$ & SNR & BER \\
\hline \multirow{3}{*}{ QPSK } & $\mathrm{Cb}(96,48)$ & $\mathrm{Cb}(384,192)$ & 2.5 & $2.2999 \times 10^{-5}$ \\
\hline & $\mathrm{Cb}(192,96)$ & $\mathrm{Cb}(768,384)$ & 2 & $1.9999 \times 10^{-6}$ \\
\hline & $\mathrm{Cb}(240,120)$ & $\mathrm{Cb}(960,480)$ & 1.5 & $1.2999 \times 10^{-5}$ \\
\hline \multirow[t]{3}{*}{ 16-QAM } & $\mathrm{Cb}(144,72)$ & $\mathrm{Cb}(576,288)$ & 7 & 0.000149 \\
\hline & $\mathrm{Cb}(192,96)$ & $\mathrm{Cb}(768,384)$ & 7 & $3.1999 \times 10^{-5}$ \\
\hline & $\mathrm{Cb}(240,120)$ & $\mathrm{Cb}(960,480)$ & 7 & $2.9998 \times 10^{-6}$ \\
\hline 64-QAM & $\mathrm{Cb}(48,24)$ & $\mathrm{Cb}(192,96)$ & 15 & $2.3 \times 10^{-5}$ \\
\hline
\end{tabular}

TABLE IV. ShOWs SimUlation Results IN COMPARISON TO HYBRID SyStem LDPC RATE 1/3

\begin{tabular}{|c|c|c|c|c|}
\hline $\begin{array}{c}\text { Modulation } \\
\text { Type }\end{array}$ & $\begin{array}{c}\text { Outer Parallel } \\
\text { Two LDPC Codes }\end{array}$ & $\begin{array}{c}\text { Inner LDPC } \\
\text { Codes }\end{array}$ & SNR & BER \\
\hline \multirow{3}{*}{ QPSK } & $\mathrm{Cb}(144,48)$ & $\mathrm{Cb}(864,288)$ & 0 & $1.1 \times 10^{-5}$ \\
\hline & $\mathrm{Cb}(288,96)$ & $\mathrm{Cb}(1728,576)$ & -0.6 & $6.9998 \times 10^{-6}$ \\
\hline & $\mathrm{Cb}(360,120)$ & $\mathrm{Cb}(2160,720)$ & -0.8 & $5.9995 \times 10^{-6}$ \\
\hline \multirow{4}{*}{ 16-QAM } & $\mathrm{Cb}(72,24)$ & $\mathrm{Cb}(432,144)$ & 6 & $2.1 \times 10^{-5}$ \\
\hline & $\mathrm{Cb}(216,72)$ & $\mathrm{Cb}(1296,432)$ & 4 & $5.2 \times 10^{-5}$ \\
\hline & $\mathrm{Cb}(288,96)$ & $\mathrm{Cb}(1728,576)$ & 4 & $8.9997 \times 10^{-6}$ \\
\hline & $\mathrm{Cb}(360,120)$ & $\mathrm{Cb}(2160,720)$ & 4 & $9.9992 \times 10^{-7}$ \\
\hline \multirow[b]{3}{*}{ 64-QAM } & $\mathrm{Cb}(72,24)$ & $\mathrm{Cb}(432,144)$ & 10 & $6 \times 10^{-6}$ \\
\hline & $\mathrm{Cb}(144,48)$ & $\mathrm{Cb}(864,288)$ & 9 & $9.9997 \times 10^{-7}$ \\
\hline & $\mathrm{Cb}(216,72)$ & $\mathrm{Cb}(1296,432)$ & 8 & $1.1 \times 10^{-5}$ \\
\hline
\end{tabular}




\section{CONCLUSIONS}

The proposed hybrid system consists of serial concatenation between parallel and serial LDPC codes. The work discusses the different generated irregular LDPC codes. From the simulation, there are two main results; the first result represents the increasing length of LDPC code which shows enhancement in system BER performance as shown in Fig. 3 to 8. The second result is obtained by using LDPC codes of code rate $1 / 3$ instead of rate $1 / 2$ which shows more improvement in system BER performance. This improvement is realized with increased system complexity. However, the designed system is a compromise between cost and performance. Hence, 16-QAM at $7 \mathrm{~dB}$ reaches $10^{-5}$ BER and QPSK $-2 \mathrm{~dB}$ which shows negative performance. Where 16-QAM reaches $10^{-6}$ at $4 \mathrm{~dB}$ SNR value. The system looks complicated, but it should be noted that the design uses a short length of irregular LDPC codes as maximum $\mathrm{C}_{\mathrm{b}}(2160,720)$ for rate $1 / 3 \mathrm{LDPC}$ as an inner encoder. The choice between different hybrid systems introduced in this work comes with two considerations, i.e., the performance and system complexity. The proposed hybrid system could be achieved in a practical application using FPGA. Since the LDPC codes show flexibility in the implementation using such technology. The LDPC decoder algorithms provide simple decoding estimation such as Bit Flipping decoding algorithm. Such consideration can reduce the hybrid system complexity.

\section{REFERENCES}

[1] G David Forny JR, "Concatenated Codes", Massachusetts Institute of technology research laboratory of electronics Cambridge, technical report 440, December 1965.

[2] Robert G. Gallager, "Low-Density Parity-Check Codes", Cambridge Mass, July 1963.

[3] Deepak Mishra, T.V.S Ram etc, "Concatenated Convolutional Codes for Deep Space Mission", International Journal of Information and Communication Technology Research, Volume 2 No. 6, June 2012.

[4] Kattaswamy Mergu, "Performance Analysis of Reed-Solomon Codes Concatenated with Convolutional Codes over AWGN Channel", Wolaita Sodo University, January 2016.
[5] Z. Shi, C. Fu, and S. Li, "Serial Concatenation and Joint Iterative Decoding of LDPC Codes and Reed-Solomon Codes", Open Research Fund of National Mobile Communication Research Laboratory, Southeast University. 2005.

[6] Satoshi Tajima, Takumi Takahashi, etc. "Iterative Decoding Based on Concatenated Belief Propagation for CRC-Aided Polar Codes", Proceedings, APSIPA Annual Summit and Conference 2018.

[7] M.Srinivasa Rao, G.Vijaya Kumar, and P.Rajesh Kumar, "Optimized BER Performance of Asymmetric Turbo Codes over AWGN Channel", International Journal of Computer Applications(0975 - 8887).Vol. 81 No.12, November 2013.

[8] Latifa Mostari and Abdelmalik Taleb-Ahmed. "Non-Binary Serial Turbo LDPC Codes Combined with High Order Constellations", Pertanika J. Sci. \& Technol. 27 (1): 33 - 47, 2019.

[9] Amjad Ali Jassim, Wael A. Hadi, and Muhanned Alfiras, "Serially Concatenated Low-density Parity Check Codes as Compatible Pairs", International Journal of Engineering \& technology, Vol.7, No 4.15 (2018)

[10] Nguyen Tung Hung, Nguyen Van Duan, Do Quoc Trinh, "Parallel and Serial LDPC Decoders for Wifi and Wimax Receivers", Chuyên san Công Singhê thông tin và Truyên thông - Sè (4-2015).

[11] Mohanad Alfiras1,*, Wael A. H. Hadi2 and Amjad Ali Jassim3, "Parallel Concatenation of LDPC Codes with LTE Modulation Schemes", Applied Mathematics \& Information Sciences An International Journal, Appl. Math. Inf. Sci. 12, No. 6, 1165-1176 (2018).

[12] Carlo Condo, "Concatenated Turbo/LDPC Codes for Deep Space Communications: Performance and Implementation", The Fifth International Conference on Advances in Satellite and Space Communications, SPACOMM, January 2013.

[13] Shwetha n, Nagaraj p, J V Narasimham," Design \& Implementation of Concatenated Turbo/LDPC Codes for Deep Space Communications", International Journal of Industrial Electronics and Electrical Engineering, ISSN: 2347-6982, Vol.3, Issue-9, pp 6-9, Sept.-2015.

[14] Dr Houman Zarrinkoub, "Understanding LTE with MATLAB from Mathematical Modeling to Simulation and Prototyping", John Wiley \& Sons, Ltd, 2014.

[15] Christopher Cox, "An Introduction to LTE LTE, LTE-Advanced, SAE, VoLTE and 4G Mobile Communications", second edition, John Wiley \& Sons, Ltd, 2014.

[16] Kun Cheng, etc. "Multi-Code-Rate Correction Technique with IR-QCLDPC: An application to QKD", 21st IEEE real time conference colonial Williamsburg, 13 mar 2019. 